\title{
Traumatic intratendinous flexor digitorum profundus rupture: A case report
}

\author{
Ashok Krishnamurthy MD, Dinakar Golla MD, WP Andrew Lee MD, J Peter Rubin MD
}

\begin{abstract}
A Krishnamurthy, D Golla, WPA Lee, JP Rubin. Traumatic intratendinous flexor digitorum profundus rupture: A case report. Can J Plast Surg 2005;13(3):151-152.

Intratendinous ruptures of a flexor digitorum profundus tendon are rare in patients who do not have rheumatoid arthritis. A case of a patient with no history of autoimmune disease who suffered a traumatic rupture of the flexor digitorum profundus tendon to the ring finger in the mid-palm is reported.
\end{abstract}

\section{Rupture traumatique du flexor digitorum profundus intratendineux : Rapport de cas}

\begin{abstract}
Les ruptures traumatiques du flexor digitorum profundus intratendineux sont rares chez les patients qui ne souffrent pas de polyarthrite rhumatoïde. On présente ici le cas d'un homme ne présentant aucun antécédent de maladie auto-immune qui a subi une rupture traumatique du flexor digitorum profundus intratendineux de l'annulaire, au niveau du milieu de la paume.
\end{abstract}

Key Words: FDP; Tendon avulsion; Traumatic rupture

A5-year-old, left hand dominant male construction worker Apresented to the emergency room after a $4.6 \mathrm{~m}$ fall from a ladder. He reported that he felt a 'pop' as he attempted to grasp the ladder with his dominant left hand during the fall. The patient had no history of previous hand trauma or arthritis. His medical history was remarkable for morbid obesity. He did not smoke tobacco and had no history of steroid use. On physical examination, he was unable to actively flex the distal phalanx of his left ring finger (Figure 1). The distal phalanx could be passively flexed through its full range. There were no lacerations present and the skin of the hand appeared normal. No area of tenderness or ecchymosis was detected in the palm. $\mathrm{X}$-rays showed no fracture or bony abnormality of the left hand. He was taken to the operating room with a diagnosis of traumatic flexor digitorum profundus (FDP) tendon avulsion.

Anticipating that the site of injury would be the insertion of the FDP at the distal phalanx, attention was focussed on this anatomical region. Under tourniquet control, a Brunner incision was made to expose flexor tendon sheath. On exploration, the FDP insertion on the distal phalanx was found to be intact (Figure 2). Next, an incision was made in the distal palmar crease over the head of the fourth metacarpal, and the flexor tendons of the fourth digit were exposed. The FDP was found to be ruptured within the palm (zone III) (Figure 2). Attempts to retrieve the proximal end of the FDP with a tendon grasper were unsuccessful, and open exposure of the carpal tunnel was required to locate this structure. The proximal and distal ends of the FDP were debrided with a number 11 blade. The tendon was then repaired with a 3-0 braided nylon core suture (modified Kessler) and a 6-0 prolene epitendinous suture. There were no complications.

An active extension/passive flexion protocol was instituted on postoperative day 2 and continued for six weeks. Three months after surgery, the patient had $0^{\circ}$ to $30^{\circ}$ of active motion at the distal interphalangeal joint (Figure 3).

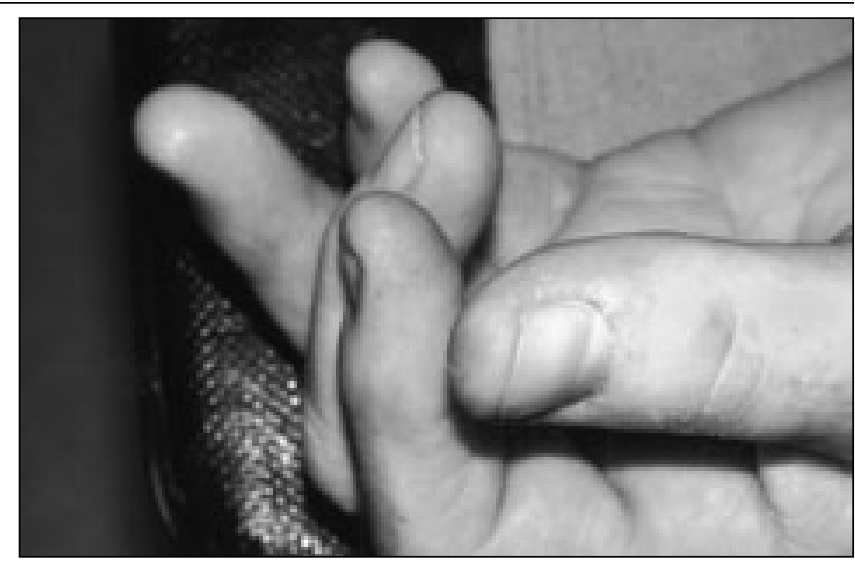

Figure 1) Preoperative photograph demonstrating the patient's inability to flex the distal interphalangeal joint of the left ring finger

\section{DISCUSSION}

While a traumatic avulsion of the FDP from its insertion at the distal phalanx is a common entity (also called Jersey Finger), this tendon usually does not rupture along its body unless weakened by a pathological condition (1). Excessive force applied to a normal flexed digit tends to result in a tear at weaker areas in the mechanical pathway, such as the musculotendinous junction, insertion at the bone or within the muscle before a tear occurs within the substance of the tendon itself (2). However, pathological states may attenuate tendon tensile strength and result in intratendinous rupture. These conditions include past trauma, bony abnormalities such as hook of hamate fractures $(3,4)$, infection, gout, tumour (5) and changes secondary to steroid treatment (6).

Intratendinous rupture of the FDP was first reported in 1891 by von Zander (1). McMaster (2) noted in 1933 that rupture of flexor tendons may occur after Colles fracture. There is a 


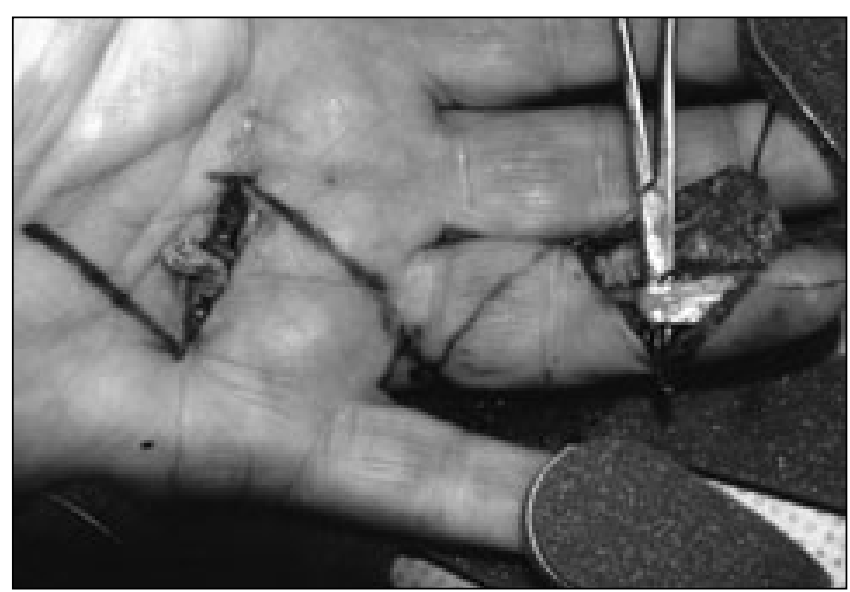

Figure 2) Intraoperative photograph showing an intact flexor digitorum profundus in zone I and distal end of the ruptured tendon in zone III

report of spontaneous rupture of flexor tendons in two patients by Mason (7) in 1930; however, it predates the recognized association of intratendinous rupture and rheumatoid arthritis published by Kersley in 1948 (8). A series of 80 flexor tendon ruptures in 78 patients reported by Boyes et al (3) in 1960 noted that only three patients had no identifiable causes. They suggested that the term 'spontaneous rupture' be reserved for those tendon ruptures that occur within the substance of the tendon, and have no identifiable or associated pathological change. Indeed, the rarity of this disorder is underscored by a 16-year experience at Cleveland Clinic in which 12 flexor tendon ruptures were treated in 10 patients without rheumatoid arthritis. All ruptures in this series were associated with underlying pathological changes (9).

A few smaller series and isolated case reports highlight the anatomical location and treatment of true spontaneous intratendinous rupture. Imbriglia and Goldstein (10) in 1987 presented a series of 10 patients who had spontaneous rupture of the FDP of the small finger. Seven of the 10 patients were treated surgically and the ruptures were found distal to the A2 pulley in two patients, in the palm in four, and in the carpal tunnel in one (10). In 1995, Naam (11) discussed a series of 13 patients seen in his practice from 1984 to 1992 . Twelve ruptures were observed to occur in zone III at the level of the lumbrical origin, and one distal to the A2 pulley. All were repaired and had good to excellent results postoperatively. Berglund and Newport (12) reported an isolated case of spontaneous rupture of tendons to both the long and ring finger profundus tendons located in zone II of a patient secondary to a grand mal seizure. There are isolated reports of ruptures throughout the literature; however, most have predisposing conditions (13-15).

In the present case report, the history and physical examination suggested that the patient had an avulsion of the FDP from the distal phalanx. The absence of an avulsed segment of bone on plain radiograph may cause one to question the diagnosis; however, it is important to remember that all type I and type II injuries, as defined by Leddy and Packer (1), are FDP avulsions without associated bony fragment. Another clue to the location of the injury may sometimes be gleaned from the patient's description of a 'popping' sensation and the presence of point tenderness. However, our patient could not localize the 'popping' sensation and did not manifest tenderness or

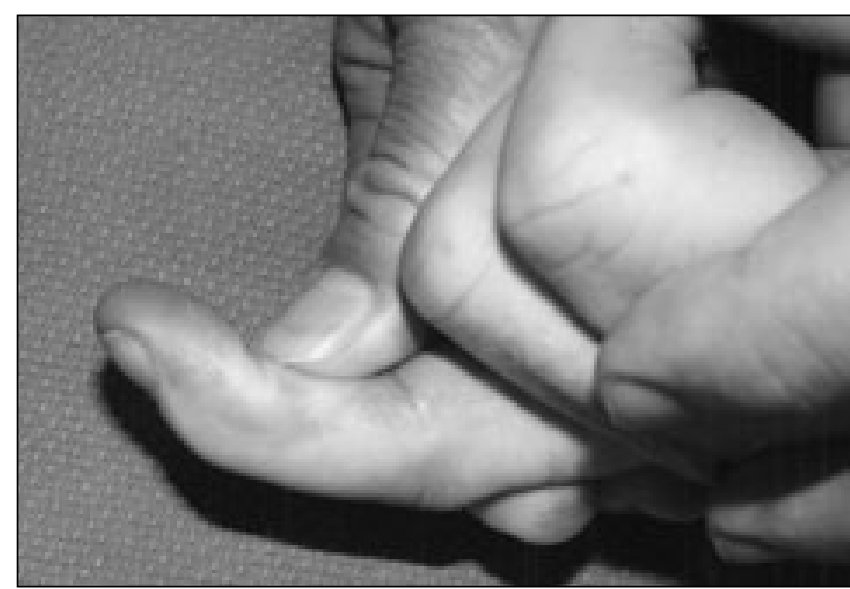

Figure 3) Postoperative photograph at three months demonstrating the patient's ability to flex the distal interphalangeal joint of the left ring finger

ecchymosis on examination. The intraoperative finding of an intact FDP insertion, as observed with our patient, should prompt the surgeon to search for a more proximal lesion. Consistent with reports by Imbriglia and Goldstein (10) and Naam (11), showing the greatest frequency of spontaneous intratendinous rupture in zone III, our patient was found to have ruptured the FDP in the mid-palm. While intratendinous rupture of flexor tendons without underlying pathological cause is uncommon, surgeons may encounter this entity when treating what appears to be a Jersey Finger and should embark on a systematic exploration of the involved tendon unit.

\section{REFERENCES}

1. Leddy JP, Packer JW. Avulsion of the profundus tendon insertion in athletes. J Hand Surg [Am] 1977;2:66-9.

2. McMaster PE. Tendon and muscle ruptures, clinical and experimental studies on the causes and location of subcutaneous ruptures. J Bone Joint Surg 1933;15:705-22.

3. Boyes JH, Wilson JN, Smith JW. Flexor-tendon ruptures in the forearm and hand. Am J Orthop 1960;42A:637-46.

4. Crosby EB, Linscheid RL. Rupture of the flexor profundus tendon of the ring finger secondary to ancient fracture of the hook of the hamate. Review of the literature and report of two cases. J Bone Joint Surg Am 1974;56:1076-8

5. Ogunro O. Avulsion of flexor profundus, secondary to enchondroma of the distal phalanx. J Hand Surg [Am] 1983;8:315-6.

6. McMaster PE. Late rupture of extensor and flexor pollicis longus tendon following colles' fractures. J Bone Joint Surg. 1932;14:93-101.

7. Mason ML. Rupture of the tendons of the hand: With a study of extensor tendon insertion. Surg Gynecologic and Obstetrics 1930;50:611-24.

8. Kersley GD. Spontaneous rupture of muscle as a complication of rheumatoid arthritis. Br Med J 1948;2:942.

9. Folmar RC, Nelson CL, Phalen GS. Ruptures of the flexor tendons in hands of non-rheumatoid patients. J Bone Joint Surg Am 1972;54:579-84.

10. Imbriglia JE, Goldstein SA. Intratendinous ruptures of the flexor digitorum profundus tendon of the small finger. J Hand Surg [Am] 1987;12:985-91.

11. Naam NH. Intratendinous rupture of the flexor digitorum profundus tendon in zones II and III. J Hand Surg [Am] 1995;20:478-83.

12. Berglund HT, Newport ML. Flexor digitorum profundus ruptures secondary to grand mal seizure. J Hand Surg [Am] 1993;18:652-4.

13. Wong FY, Pho RW. Median nerve compression, with tendon ruptures, after Colles' fracture. J Hand Surg [Br] 1984;9:139-41.

14. Kumar S, James R. Closed rupture of flexor profundus tendon in the palm. J Hand Surg [Br] 1985;10:193-4.

15. Takami H, Takahashi S, Ando M. Rupture of the flexor digitorum profundus tendon in the palm caused by repeated, chronic direct trauma. J Hand Surg [Am] 1993;18:65-7. 